\title{
ESTABILIZAÇÃO DE SISTEMAS DESCRITORES POR REALIMENTAÇÃO DE SAÍDAS VIA SUBESPAÇOS INVARIANTES
}

\author{
Eugênio B. Castelan* \\ eugenio@das.ufsc.br \\ * Departamento de Automação e Sistemas \\ Universidade Federal de Santa Catarina \\ 88040-900, Florianópolis, SC, Brasil.
}

\begin{abstract}
We consider the problem of strong stabilization of descriptor systems via output feedback. The proposed results are based on the concept of $(C, A, E, B)$-invariance and its algebraic characterization by coupled Sylvester equations and coupled Lyapunov-like equations. Based either on eigenstructure assignment techniques or on the solution of linear matrix inequalities and equalities, we propose algorithms for computation of the output feedback matrix for descriptor systems that verify some conditions. The proposed results and algorithms generalize previous works developed for classical linear systems and for particular classes of descriptor systems.
\end{abstract}

KEYWORDS: Descriptor systems, Output feedback, Sylvester equations, Lyapunov equations, LMI/LMEs.

\section{RESUMO}

Considera-se o problema da existência e do cálculo de uma matriz de realimentação de saídas que estabiliza fortemente um sistema descritor linear. Os resultados apresentados baseiam-se no conceito de $(C, A, E, B)$-invariância e de sua caracterização algébrica através de equações acopladas de Sylvester e de equações acopladas de tipo Lyapunov. Apresentam-se algoritmos para o cálculo da matriz de realimentação de saídas, baseados em posicionamento de autoes-

Artigo submetido em 05/08/04

1a. Revisão em 21/02/05

2a. Revisão em 03/10/05

Aceito sob recomendação do Ed. Assoc. Prof. Liu Hsu trutura ou na solução de desigualdades e igualdades matriciais lineares. Os resultados e algoritmos apresentados generalizam resultados anteriores propostos para sistemas lineares clássicos e para classes particulares de sistemas descritores lineares.

PALAVRAS-CHAVE: Sistemas descritores, Realimentação de saídas, Equações de Sylvester, Equações de Lyapunov, LMI/LMEs.

\section{INTRODUÇÃO}

A estabilização de sistemas lineares por realimentação estática de saídas é, reconhecidamente, um problema fundamental em teoria de controle. Isto decorre do fato que em grande parte das aplicações práticas apenas parte da informação dos estados do sistema estar disponível a partir das saídas medidas e, além disso, a obtenção de um controlador de complexidade mínima ser um requisito importante.

Considera-se neste artigo o problema de estabilização por realimentação estática de saídas de sistemas descritores lineares. Este tipo de sistemas aparece naturalmente na modelagem de vários sistemas físicos como circuitos elétricos, sistemas de potência e outros sistemas interconectados, sistemas robóticos e sistemas econômicos (Dai, 1989; Verghese et al., 1981; Lewis, 1986). Basicamente, um sistema descritor de ordem $n$ consiste de uma mistura de $q$ equações diferenciais e $(n-q)$ equações algébricas (Dai, 1989). Devido a esta característica, o controle de sistemas descritores requer, além da garantia da estabilidade assintótica em ma- 
lha fechada, outras duas propriedades importantes: a regularidade e a ausência de modos impulsivos. Um sistema em malha-fechada que satisfaz estas três propriedades é dito ser fortemente estável, ou simplesmente $S$-estável (do inglês "strongly stable") (Varga, 1995).

Nos últimos anos, diversos trabalhos sobre a estabilização de sistemas descritores por realimentação estática de saídas têm sido propostos, aplicando-se diferentes técnicas para considerar as características específicas destes sistemas. Pode-se destacar, particularmente, vários resultados baseados em técnicas de posicionamento de pólos e autoestrutura (Fletcher, 1988; Duan, 1995; Duan, 1999; Castelan et al., 2001; Llanos Villarreal et al., 2000; Castelan et al., 2002; Duan, 2004), resultados baseados na teoria de estabilidade de Lyapunov (Zhang et al., 2005; Kuo and Fang, 2003), assim como alguns trabalhos que consideram a utilização de realimentação estática de saídas para a solução de outros problemas de controle (Barbosa and Trofino, 2003; Duan et al., 2000; Chu, 2001).

Sem ser exaustiva, a bibliografia acima citada (veja também suas referências) revela o interesse da comunidade científica, nacional e internacional, no tratamento de problemas de controle de sistemas descritores por realimentação estática de saídas. Assim, dentro do contexto anteriormente descrito, considera-se neste artigo uma abordagem que explora propriedades estruturais e geométricas do sistemas a controlar e utiliza equações generalizadas de Sylvester ou de Lyapunov para a caracterização e o cálculo de soluções.

As linhas gerais da abordagem proposta podem ser resumidas como segue. Note, inicialmente, que a estabilidade forte pode ser interpretada em termos da autoestrutura do sistema em malha fechada: (i) a estabilidade assintótica é equivalente a que todos os pólos finitos estejam no interior do semiplano esquerdo do plano complexo; (ii) a ausência dos modos impulsivos é equivalente a ter $q$ pólos finitos em malha fechada; e (iii) a regularidade é garantida se o sistema é livre de modos impulsivos. Baseando-se nesta interpretação, condições necessárias e suficientes para a existência de uma realimentação de saídas $S$-estabilizável são estabelecidas a partir de um conjunto de equações generalizadas acopladas de Sylvester (Fletcher, 1988; Duan, 1999; Castelan et al., 2001). Como no caso de sistemas puramente diferenciais (também chamados de sistemas normais (Dai, 1989)), as equações de Sylvester podem ser interpretadas a partir de conceitos da teoria de controle geométrico, ou, mais especificamente, utilizando-se a noção de subespaços $(C, A, E, B)$ invariantes. Além disso, mostra-se que equações generalizadas de tipo Lyapunov também podem ser usadas para descrever a $(C, A, E, B)$-invariância de subespaços e, portanto, para obter um conjunto de condições necessárias e suficientes para a existência de uma realimentação de saídas que estabiliza fortemente o sistema.
Os resultados citados permitem propor uma extensão para o caso de sistemas descritores dos procedimentos algorítmicos em dois passos relatados em (Castelan et al., 2003) para a estabilização de sistemas normais. Diferentemente de resultados propostos anteriormente em (Llanos Villarreal et al., 2000; Castelan et al., 2002), nenhuma estrutura particular é requerida das matrizes que definem o sistema descritor em malha aberta. Assim, sob hipóteses de estabilizabilidade e de detectabilidade do sistema a controlar, necessárias para existência de solução do problema, propõem-se algoritmos para estabilização de sistemas descritores que satisfaçam uma condição que relaciona o número de entradas e saídas com o número de variáveis algébricas do sistema, a qual pode ser considerada uma generalização da conhecida condição de Kimura para sistemas normais (Syrmos and Lewis, 1993) . Importante salientar o fato que também no caso de sistemas descritores, a solução do primeiro passo do procedimento proposto pode ser associada a um sistema normal de ordem reduzida, o qual pode ser obtido aplicando-se a seqüência de transformações de coordenadas relatadas no presente artigo (Castelan and da Silva, 2005).

O artigo tem a organização seguinte. Na seção 2, apresentase o problema a ser tratado, bem como algumas definições e notações básicas associadas a sistemas descritores. Na seção 3 apresentam-se as condições de existência de solução do problema de $S$-estabilização, através das equações generalizadas acopladas de Sylvester e de Lyapunov. Baseandose nos algoritmos apresentados em (Castelan et al., 2003), propõe-se na seção 4 um algoritmo para resolver o problema de estabilização forte via posicionamento de autoestrutura. $\mathrm{O}$ algoritmo baseado na solução de desigualdades e igualdades matriciais lineares (LMI/LMEs) é apresentado na seção 5. Um exemplo ilustrativo e comentários concluem o artigo.

Notação: Para $M \in \Re^{n \times m}$, sua Imagem e Espaço Nulo são dados por $\operatorname{Im}(M)=\left\{b \in \Re^{n} ; M x=b \operatorname{com} x \in \Re^{m}\right\}$ e $\operatorname{Ker}(M)=\{x \in$ $\left.\Re^{m} ; M x=0\right\}$, respectivamente. $\sigma(A)$ denota conjunto de autovalores, ou espectro, da matriz $A \in \Re^{n \times n}$. Se $P=P^{\prime} \in \Re^{n \times n}$, então $P>0(P \geq$ $0)$ signifi ca que $P$ é uma matriz defi nida (semidefi nida) positiva. $I$ é a matriz identidade de dimensão $n$. Relativamente ao conjunto de números complexos $\mathcal{C}$, tem-se $\mathcal{C}^{-}=\{\alpha+j \beta \in \mathcal{C} ; \alpha<0\}$ e $\overline{\mathcal{C}}^{+}=\{\alpha+j \beta \in$ $\mathcal{C} ; \alpha \geq 0\}$. A dimensão de um subespaço $\mathcal{V} \subset \mathcal{X}$ é denotada $\operatorname{dim}\{\mathcal{V}\}$. Se $\mathcal{V}$ é subespaço $A$-invariante, i.e.: $A \mathcal{V} \subset \mathcal{V}$, então $A \mid \mathcal{V}$ é a restrição de $A$ em $\mathcal{V}$ e $A \mid(\mathcal{X} / \mathcal{V})$ é o mapa induzido por $A$ no subespaço quociente $\mathcal{X} / \mathcal{V}$.

\section{FORMULAÇÃO DO PROBLEMA E PRE- LIMINARES}

Seja um sistema descritor linear e invariante no tempo descrito por:

$$
\begin{aligned}
E \dot{x}(t) & =A x(t)+B u(t) \\
y(t) & =C x(t)
\end{aligned}
$$


em que: $x \in \mathcal{X} \sim \Re^{n}, u \in \mathcal{U} \sim \Re^{m}, y \in \mathcal{Y} \sim \Re^{p} \mathrm{e}$ $E \in \Re^{n \times n}$, posto $(E)=q<n$; as demais matrizes têm dimensões adequadas com posto $(B)=m$ e posto $(C)=p$. Considera-se que $p<q$.

O problema a ser tratado consiste em encontrar uma lei de controle do tipo realimentação de saídas

$$
u(t)=K y(t), K \in \Re^{m \times p}
$$

tal que o sistema em malha fechada

$$
E \dot{x}(t)=(A+B K C) x(t)
$$

seja regular, assintoticamente estável e livre de modos impulsivos (Varga, 1995). O problema de cálculo de uma realimentação de saídas tal que essas propriedades são verificadas em malha fechada, será denominado de estabilização forte ou, simplesmente, S-estabilização (do inglês "Strong Stabilization”).

Relativamente ao sistema em malha-fechada, consideram-se as definições básicas e notações seguintes (Dai, 1989; Varga, 1995). O sistema descritor (4) é regular se $\operatorname{det}(\lambda E-A-$ $B K C) \neq 0$. Sob a hipótese de regularidade, o conjunto de pólos do sistema (finitos e infinitos) representa o conjunto de autovalores generalizados do $\operatorname{par}(E, A+B K C)$. O conjunto de seus pólos finitos, representado por $\sigma(E, A+B K C)$, é formado pelas raízes da equação característica $\operatorname{det}(\lambda E-$ $A-B K C)=0$. Se o número de pólos finitos é menor que $\operatorname{posto}(E)=q$, então o sistema descritor possui modos impulsivos. O sistema é livre de modos impulsivos se ele tem exatamente $q$ pólos finitos. O sistema descritor é assintoticamente estável se todos os seus pólos finitos pertencem a $\mathcal{C}^{-}$, o semiplano esquerdo aberto do plano complexo. Então, o sistema (4) é fortemente estável (regular, assintoticamente estável e livre de modos impulsivos) se, e somente se, existem matrizes $V_{f} \in \Re^{n \times q}, T_{f} \in \Re^{q \times n}$ e $H_{f} \in \Re^{q \times q}$, com $\sigma\left(H_{f}\right) \in \mathcal{C}^{-}$, tais que (Duan, 1995):

$$
\begin{aligned}
(A+B K C) V_{f} & =E V_{f} H_{f} \\
T_{f}(A+B K C) & =H_{f} T_{f} E \\
T_{f} E V_{f} & =I_{q} \\
\text { posto }\left(E V_{f}\right) & =\text { posto }\left(T_{f} E\right)=q
\end{aligned}
$$

em que $\sigma\left(H_{f}\right)$ corresponde a $\sigma(E, A+B K C)$. Observe nestas equações que se considerarmos $H_{f}$ sob a forma real de Jordan, as colunas das matrizes $V_{f}$ e $T_{f}$ serão formadas a partir dos autovetores generalizados (reais) à direita e à esquerda, respectivamente, associados aos pólos finitos do sistema em malha-fechada.

A estabilizabilidade e a detectabilidade são requisitos fundamentais para a solução do problema considerado. Então, por hipótese, considera-se que o sistema representado por
( $E, A, B)$ é fortemente estabilizável ou, equivalentemente (Bunse-Gerstner et al., 1992; Varga, 1995):

$$
\begin{gathered}
\operatorname{posto}\left(\left[\begin{array}{cc}
\lambda E-A & B
\end{array}\right]\right)=n, \forall \lambda \text { finito } \in \overline{\mathcal{C}}^{+} \\
\text {posto }\left(\left[\begin{array}{lll}
E & A R_{\infty} & B
\end{array}\right]\right)=n
\end{gathered}
$$

em que $R_{\infty} \in \Re^{n \times n-q}$, com posto $\left(R_{\infty}\right)=(n-q)$, é tal que $E R_{\infty}=0$. Dualmente, considera-se que o sistema representado por $(C, A, E)$ é fortemente detectável:

$$
\begin{gathered}
\text { posto }\left(\left[\begin{array}{c}
\lambda E-A \\
C
\end{array}\right]\right)=n, \forall \lambda \text { finito } \in \overline{\mathcal{C}}^{+} \\
\text {posto }\left(\left[\begin{array}{c}
E \\
L_{\infty} A \\
C
\end{array}\right]\right)=n
\end{gathered}
$$

em que $L_{\infty} \in \Re^{n-q \times n}$, com posto $\left(L_{\infty}\right)=(n-q)$, é tal que $L_{\infty} E=0$. Caso as condições (9) e (11) sejam válidas para qualquer $\lambda \in \mathcal{C}$, então $(E, A, B)$ é fortemente controlável e $(C, A, E)$ é fortemente observável, respectivamente.

Observação 1 Ao longo deste trabalho, considera-se a possibilidade do par $(E, A)$, associado ao sistema em malha aberta, ser não-regular, ou seja: $\operatorname{det}(\lambda E-A)=0, \forall \lambda \in \mathcal{C}$. Isto ocorre somente se $A$ é singular. Então, devido a possibilidade de existência da intersecção entre subespaços $\mathcal{N}=$ $\operatorname{Ker}(E) \cap \operatorname{Ker}(A)$, pode-se distinguir dois casos de sistemas não-regulares:

Caso i) $\mathcal{N}=\{0\} \Longrightarrow d=\operatorname{dim}\{\mathcal{N}\}=0$;

Caso ii) $\mathcal{N} \neq\{0\} \Longrightarrow d=\operatorname{dim}\{\mathcal{N}\} \geq 1$.

No segundo caso, tem-se: $(\lambda E-A) \eta=0, \forall \eta \in \operatorname{Ker}(\mathcal{N})$. Observe que se $(E, A)$ é regular, tem-se $d=0$, como no caso $i$ ) acima.

Complementarmente, apresentam-se a seguir alguns conceitos de controle geométrico associados ao sistema descritor (1)-(2) (Ozcaldiran and Lewis, 1990) (Castelan et al., 2001), utilizados na sequiência para a interpretação de alguns resultados. Esses conceitos são uma extensão para sistemas descritores dos conceitos introduzidos em (Syrmos and Lewis, 1993) para o caso de sistemas normais.

Um subespaço $\mathcal{V} \subset \mathcal{X}$ é $(A, E, B)$-invariante se existe $F: \mathcal{X} \longrightarrow \mathcal{Y}$ tal que $(A+B F) \mathcal{V} \subset E \mathcal{V}$, ou equivalentemente, $A \mathcal{V} \subset E \mathcal{V}+\operatorname{Im}(B)$. Dualmente, um subespaço $\mathcal{T} \subset \mathcal{X}$ é $(C, A, E)$-invariante se existe $L: \mathcal{Y} \longrightarrow \mathcal{X}$ tal que $(A+L C) \mathcal{T} \subset E \mathcal{T}$, ou equivalentemente, $E \mathcal{T} \supset$ $A(\mathcal{T} \cap \operatorname{Ker}(C))$.

Definição $1 \mathrm{Um}$ subespaço $\mathcal{V} \subset \mathcal{X}$, de dimensão- $v$, é $(C, A, E, B)$-invariante se $\mathcal{V}$ é $(A, E, B)$-invariante e $(C, A, E)$-invariante. 
As propriedades de estabilizabilidade e detectabilidade podem ser consideradas através das duas definições a seguir: (i) um subespaço $(A, E, B)$-invariante $\mathcal{V}$ é $(A, E, B)$ internamente estabilizável se existe $F$ tal que $(E, A+B F) \mid \mathcal{V}$ é (assintoticamente) estável;

(ii) um subespaço $(C, A, E)$-invariante $\mathcal{V}$ é $(C, A, E)$ externamente detectável se existe $L$ tal que $(E, A+$ $L C) \mid \mathcal{X} / \mathcal{V}$ é (assintoticamente) estável.

A partir das definições $(i)$ e $(i i)$, e considerando a propriedade de regularizabilidade (Ozcaldiran and Lewis, 1990), tem-se ${ }^{1}$ :

Definição 2 Um subespaço $\mathcal{V} \subset \mathcal{X}$, de dimensão $v$, é fortemente $O . S$. $(C, A, E, B)$-invariante se $\mathcal{V}$ é $(A, E, B)$ internamente estabilizável e $(C, A, E)$-externamente detectável e, além disso, $\operatorname{Ker} E \cap \mathcal{V}=\{0\}$.

Seja $V \in \Re^{n \times v}$ tal que $\operatorname{Im}(V)=\mathcal{V}$. Então a condição $\operatorname{Ker}(E) \cap \mathcal{V}=\{0\}$ pode ser equivalentemente substituída por (Ozcaldiran and Lewis, 1990):

$$
\operatorname{posto}(E V)=v
$$

Considerando que (13) é verificada, a matriz $T \in \Re^{(q-v) \times n}$ é um anulador à esquerda de $E V$, se $T E V=0$ e $\operatorname{Ker}(T) \cap$ $\operatorname{Im}(E)=\{0\}$. Importante observar que a um subespaço $\mathcal{V}=\operatorname{Im}(V)$ fortemente $O . S$. $(C, A, E, B)$-invariante podese associar um par de equações generalizadas de Sylvester que devem satisfazer, em particular, a condição de acoplamento anterior (Castelan et al., 2001).

Observação 2 O problema e os conceitos acima considerados, bem como os resultados que serão apresentados na seqüência, podem ser facilmente especializados para o caso de sistemas normais (Castelan et al., 2003; Syrmos and Lewis, 1993). Para tanto, basta considerar que posto $(E)=n$ ou, classicamente, $E=I_{n}$, e não levar em consideração as relações que estejam associadas à existência dos espaços nulos à direita e à esquerda da matriz $E$.

\section{REALIMENTAÇÃO DE SAÍDAS E SUBESPAÇOS O.S. $(C, A, E, B)$ - INVARIANTES}

O resultado a seguir relaciona a existência de uma realimentação de saídas que estabiliza fortemente o sistema em malha fechada à existência de soluções para duas equações acopladas de Sylvester e, portanto, ao conceito de subespaços fortemente O.S. ( $C, A, E, B)$-invariantes (Castelan et al., 2001).

\footnotetext{
${ }^{1} 0 . S$. é uma abreviação para expressão em inglês Output Stabilizable.
}

Teorema 1 Existe uma matriz $K \in \Re^{m \times p}$ tal que $\sigma(E, A+$ $B K C) \in \mathcal{C}^{-}$e o sistema em malha fechada (4) é regular e livre de modos impulsivos se e somente se para algum número inteiro positivo $v \leq q$, as seguintes condições são verificadas para matrizes $\left(V \in \Re^{n \times v}, H_{V} \in \Re^{v \times v}, W \in \Re^{m \times v}\right)$, $\left(T \in \Re^{(q-v) \times n}, H_{T} \in \Re^{(q-v) \times(q-v)}, U \in \Re^{(q-v) \times p}\right)$ :

$$
\begin{aligned}
A V-E V H_{V} & =-B W, & \sigma\left(H_{V}\right) \in \mathcal{C}^{-} \\
T A-H_{T} T E & =-U C, & \sigma\left(H_{T}\right) \in \mathcal{C}^{-} \\
T E V & =0 & \\
\operatorname{Ker}(C V) & \subseteq \operatorname{Ker}(W) & \\
\operatorname{Ker}\left(B^{\prime} T^{\prime}\right) & \subseteq \operatorname{Ker}\left(U^{\prime}\right) &
\end{aligned}
$$

$\operatorname{com} \operatorname{posto}(E V)=v$ e posto $(T E)=(q-v)$.

A demonstração proposta em (Castelan et al., 2001) utiliza, fundamentalmente, argumentos de posicionamento de autoestrutura. Em particular, ela leva em consideração que as propriedades desejadas podem ser associadas apenas à estrutura finita do sistema descritor em malha fechada, através das relações (5)-(8). Então, para efeitos de demonstração, podese considerar qualquer inteiro positivo $v \leq q$ que permita realizar o particionamento do subespaço invariante associado aos pólos finitos de (4) e utilizar, sem perda generalidade $H_{f}=\left[\begin{array}{cc}H_{V} & 0 \\ 0 & H_{T}\end{array}\right], V_{f}=\left[\begin{array}{ll}V & \bar{V}\end{array}\right]$ e $T_{f}=\left[\begin{array}{c}\bar{T} \\ T\end{array}\right]$. Além disso, é importante salientar que as condições (17) e (18) são condições equivalentes à existência de $K \in \Re^{m \times p}$ que satisfaz, conjuntamente, $K C V=W$ e $T B K=U$ (Duan, 1995; Chen, 1996).

Do ponto de vista dos conceitos geométricos introduzidos na seção anterior, as equações acopladas de Sylvester (14)(16) são equivalentes ao fato de $\mathcal{V}=\operatorname{Im}(V)$, de dimensão $v$, ser um subespaço fortemente $O . S$. $(C, A, E, B)$ invariante. Portanto, como no caso de sistemas normais (Castelan et al., 2003), o problema de $S$-estabilização de sistemas descritores pode ser associado à construção destes subespaços invariantes, como será discutido nas seções seguintes.

Apresenta-se agora uma caracterização dos subespaços fortemente $O . S$. ( $C, A, E, B)$-invariantes via equações generalizadas de Lyapunov. Esta caracterização pode ser obtida a partir das relações apresentadas no teorema anterior, ao substituirem-se as condições de estabilidade expressas nas equações de Sylvester (14)-(15) pelas duas condições de estabilidade de Lyapunov a seguir (Chen, 1996) :

$$
\begin{aligned}
\sigma\left(H_{V}\right) \in \mathcal{C}^{-} \Leftrightarrow & \forall Q_{V}=Q_{V}^{\prime}>0, \exists \Pi=\Pi^{\prime}>0 \\
& \text { tal que } \Pi H_{V}^{\prime}+H_{V} \Pi=-Q_{V} \\
\sigma\left(H_{T}\right) \in \mathcal{C}^{-} \Leftrightarrow \quad & \forall Q_{T}=Q_{T}^{\prime}>0, \exists \Gamma=\Gamma^{\prime}>0 \\
& \text { tal que } H_{T}^{\prime} \Gamma+\Gamma H_{T}=-Q_{T}
\end{aligned}
$$


Então, a partir do Teorema 1, obtém-se o resultado seguinte.

Teorema 2 : Existe uma matriz $K \in \Re^{m \times p}$ tal que $\sigma(E, A+B K C) \in \mathcal{C}^{-}$e o sistema em malha fechada (4) é regular e livre de modos impulsivos, se e somente se as condições seguintes são verificadas para algum inteiro positivo $v \leq q$ e para algum par de matrizes $V \in \Re^{n \times v}$ e $T \in \Re^{q-v \times n}$ tais que $T E V=0$, com $\operatorname{posto}(E V)=v$ e $\operatorname{posto}(T E)=(q-v)$ :

(i) $\forall Q_{V}=Q_{V}^{\prime}>0, Q_{V} \in \Re^{v \times v}$, existem matrizes $P=P^{\prime} \geq 0, P \in \Re^{n \times n}$ e $Y \in \Re^{m \times n}$ tais que:

$$
\begin{array}{r}
A P E^{\prime}+E P A^{\prime}+B Y E^{\prime}+E Y^{\prime} B^{\prime}=-E V Q_{V} V^{\prime} E^{\prime} \\
\bar{P}=V^{\prime} P V>0 ; T E P E^{\prime} T^{\prime}=0 \\
Y=W_{\Pi} V^{\prime} \text { para algum } W_{\Pi} \in \Re^{m \times v}
\end{array}
$$

(ii) $\forall Q_{T}=Q_{T}^{\prime}>0, Q_{T} \in \Re^{(q-v) \times(q-v)}$, existem matrizes $S=S^{\prime} \geq 0, S \in \Re^{n \times n}$ e $Z \in \Re^{n \times p}$ tais que

$$
\begin{aligned}
& A^{\prime} S E+E^{\prime} S A+ C^{\prime} Z^{\prime} E+E^{\prime} Z C=-E^{\prime} T^{\prime} Q_{T} T E \\
& \bar{S}=T S T^{\prime}>0 ; V^{\prime} E^{\prime} S E V=0 \\
& Z=T^{\prime} U_{\Gamma} \text { para algum } U_{\Gamma} \in \Re^{(q-v) \times p} \\
& \text { Ker } C P \subseteq \operatorname{Ker} Y \\
& \text { (iii) } \quad \operatorname{Ker} B^{\prime} S^{\prime} \subseteq \operatorname{Ker} Z^{\prime}
\end{aligned}
$$

A demonstração do resultado acima (Castelan et al., 2001) é tecnicamente similar à demonstração do Teorema $2.1 \mathrm{em}$ (Castelan et al., 2003). Pode-se também resgatar a condição de acoplamento entre as partes $(i)$ e $(i i)$ do Teorema 2 sob a forma: $S E P=0$, com posto $(S E)=q-\operatorname{posto}(E P)$.

Cabe salientar a equivalência entre as relações (21)-(23) e a equação de Sylvester (14) e, de forma similar, a equivalência entre (24)-(26) e (15). Estas equivalências mostram a possibilidade de descrever propriedades geométricas (estruturais) por meio de relações de tipo Lyapunov. Potencialmente, a descrição de tipo Lyapunov permite a extensão de métodos de análise e projeto desenvolvidos a partir das equações de Sylvester, para serem tratados numericamente através de técnicas de resolução de LMI/LMEs (Boyd et al., 1994).

\section{ASPECTOS ALGORÍTMICOS}

As condições algébricas estabelecidas nos Teoremas 1 e 2 podem ser usadas para construir subespaços fortemente $O . S$. $(C, A, E, B)$-invariantes, os quais permitirão o cálculo de realimentações de saídas estabilizadoras. Para efeitos de cálculo, considera-se $v=p$, o que permite, em particular, a obtenção da matriz de realimentação de estados a partir da relação $K C V=W$, associada à relação (17). Então, como no caso de sistemas normais (Syrmos and Lewis, 1993; Castelan et al., 2003), o cálculo da matriz $K$ pode ser realizado levando-se em conta o requisito de acoplamento e resolvendo-se subsequentemente as equações acopladas generalizadas de Sylvester ou de Lyapunov.

\subsection{Extensão do algoritmo de Syrmos- Lewis}

Baseado no algoritmo apresentado em (Syrmos and Lewis, 1993) (veja também (Castelan et al., 2003)), o procedimento básico seguinte foi proposto em (Castelan et al., 2001) para calcular uma realimentação de saídas que estabiliza o sistema em malha fechada, quando $(m+p)>q+d$, em que, conforme a Observação $1, d=\operatorname{dim}\{\operatorname{Ker}(E) \cap$ $\operatorname{Ker}(A)\}$. Os autovalores de malha fechada são posicionados arbitrariamente próximos ao conjunto $\Lambda=\left\{\Lambda_{T}, \Lambda_{V}\right\}$, formado pela união dos conjuntos simétricos de autovalores pré-especificados $\Lambda_{T}=\left\{\lambda_{1}, \ldots, \lambda_{q-p}\right\}$ e $\Lambda_{V}=$ $\left\{\lambda_{q-p+1}, \ldots, \lambda_{q}\right\}$. Para efeitos de posicionamento arbitrário de pólos, o sistema $(E, A, B, C)$ é considerado fortemente controlável e fortemente observável.

\section{Algoritmo 1}

Passo 1: Escolhe-se $H_{T} \in \Re^{(q-p) \times(q-p)}$ tal que $\sigma\left(H_{T}\right)=$ $\Lambda_{T} \in \mathcal{C}^{-}$e resolve-se a equação (15) para encontrar $T \in$ $\Re^{(q-p) \times n}$ tal que

$$
\text { posto }\left(\left[\begin{array}{c}
T E \\
C
\end{array}\right]\right)=q
$$

A condição (29) garante, em particular, que posto $(T E)=$ $q-p$.

Passo 2: Resolve-se a equação (14) para alguma matriz $H_{V} \in \Re^{p \times p}$ tal que $\sigma\left(H_{V}\right)=\Lambda_{V} \in \mathcal{C}^{-}$, tomando em consideração que $V$ deve verificar a condição de acoplamento (16) e que posto $(E V)=p$.

Passo 3: Por construção, a matriz $V$ deve verificar posto $(C V)=p$ e $K$ pode ser calculada por:

$$
K=W(C V)^{-1}
$$

Considerando-se as matrizes $H_{T}$ e $H_{V}$ sob a forma diagonal de Jordan, os dois primeiros passos podem ser resolvidos utilizando-se técnicas padrão de posicionamento de autoestrutura, como segue. No Passo 1 , calculam-se $t_{j} \in \mathcal{C}^{n}$ e $u_{j} \in \mathcal{C}^{p}$, tais que :

$$
\left[\begin{array}{ll}
t_{j}^{\prime} & u_{j}^{\prime}
\end{array}\right]\left[\begin{array}{c}
A-\lambda_{j} E \\
C
\end{array}\right]=0 \forall j=1, \ldots,(q-p)
$$

As linhas da matriz $T \in \Re^{(q-p) \times n}$, denotadas por $T_{j}$, são formadas a partir dos vetores $t_{j}$, como segue:

- se $\lambda_{j} \in \Re$, então $T_{j}=t_{j}^{\prime}$; 
- se $\lambda_{j} \in \mathcal{C}$, considera-se $\lambda_{j+1}=\lambda_{j}^{*}$ e $\left\{\begin{aligned} T_{j} & =\operatorname{Re}\left(t_{j}^{\prime}\right) \\ T_{j+1} & =\operatorname{Imag}\left(t_{j}^{\prime}\right)\end{aligned}\right.$.

Sob a condição que o sistema seja fortemente observável (detectável), é sempre possível encontrar uma solução no Passo 1 tal que a matriz $T$ verifica a condição (29). Para tanto, mostra-se no final desta seção uma sistemática para o cálculo de $T$ baseada na definição de um sistema de ordem reduzida (Castelan and da Silva, 2005).

No Passo 2, determinam-se $v_{i} \in \mathcal{C}^{n}$ e $w_{i} \in \mathcal{C}^{m}$ :

$$
\begin{gathered}
{\left[\begin{array}{cc}
A-\lambda_{i} E & B \\
T E & 0
\end{array}\right]\left[\begin{array}{c}
v_{i} \\
w_{i}
\end{array}\right]=\left[\begin{array}{l}
0 \\
0
\end{array}\right],} \\
\forall i=(q-p)+1, \ldots, q .
\end{gathered}
$$

De forma similar ao caso anterior, as matrizes $V$ e $W$ utilizadas para o cálculo de $K$ podem ser construídas somente com elementos reais.

Observe que a condição generalizada de Kimura, $(m+p)>$ $q+d$, garante que para qualquer $\lambda_{i} \in \mathcal{C}$, sempre existe um vetor $\left[v_{i}^{\prime} w_{i}^{\prime}\right]^{\prime} \neq 0$ que satisfaz (32). Entretanto, para garantir que os autovalores $\lambda_{i}$ do Passo 2 sejam livremente alocáveis, o sistema matricial $P(\lambda)=\left[\begin{array}{cc}A-\lambda E & B \\ T E & 0\end{array}\right]$ de dimensão $(n+q-p) \times(n+m)$, deve ter posto completo por linhas $\forall \lambda \mathrm{e}$ deve-se garantir a possibilidade de encontrar os vetores $v_{i}$, a partir de (32), que não pertençam a $K \operatorname{er}(E)$. Se para algum valor particular $\lambda=\bar{\lambda}$ tivermos posto $(P(\bar{\lambda}))<n+q-p$, então $\bar{\lambda}$ é um zero invariante do sistema e deve fazer parte do espectro de $H_{V}$ (e, portanto, satisfazer $\bar{\lambda} \in \mathcal{C}^{-}$). Felizmente, nos casos raros em que o cálculo de $T$ leva a $P(\lambda)$ cujo posto não é completo, uma pequena modificação dos autovalores utilizados no Passo 1 e, portanto, o cálculo de uma nova matriz $T$, são geralmente suficientes para resolver este problema e tornar viável a alocação arbitrário dos pólos no segundo passo. Este tipo de estratégia é classicamente adotada na literatura relacionada ao posicionamento de pólos por realimentação de saídas (veja, por exemplo, (Syrmos and Lewis, 1993) e comentários em (Castelan et al., 2003)).

Observação 3 Considerando-se que posto $(P(\lambda))=n+q-$ $p \forall \lambda$, e levando-se em conta a condição generalizada de Kimura, tem-se:

$$
\operatorname{dim}\{\operatorname{Ker}(P(\lambda))\}=m+p-q>d
$$

Isto garante a existência de soluções para (32), mesmo nos casos em que $(E, A)$ é não-regular. Em particular, se $d=$ $\operatorname{dim}\{\mathcal{N}=\operatorname{Ker}(E) \cap \operatorname{Ker}(A)\} \geq 1$, verifica-se a partir da Observação 1 que: $P(\lambda)\left[\begin{array}{l}\eta \\ 0\end{array}\right], \forall \eta \in \mathcal{N}$. Então, (33) garante a possibilidade de encontrar outras soluções para (32), tais que $v_{i} \notin \mathcal{N} \Rightarrow E v_{i} \neq 0$.

\subsection{Passo 1: Abordagem via sistema de ordem reduzida}

Por hipótese, considera-se que o sistema $(C, A, E)$ é fortemente detectável. Além disso, considera-se que (Castelan and da Silva, 2005):

$$
\text { posto }\left(\left[\begin{array}{c}
L_{\infty} A \\
C
\end{array}\right]\right)=n-q+p, \operatorname{com} p<q \text {. }
$$

Na seqüência, as matrizes que descrevem o sistema descritor em malha aberta serão consideradas, sem perda de generalidade, sob a forma algébrica-diferencial (Dai, 1989):

$$
\begin{array}{cc}
E=\left[\begin{array}{cc}
I_{q} & 0 \\
0 & 0
\end{array}\right], \quad A=\left[\begin{array}{ll}
J_{1} & J_{2} \\
J_{3} & J_{4}
\end{array}\right] \\
B=\left[\begin{array}{l}
B_{1} \\
B_{2}
\end{array}\right] \quad, \quad C=\left[\begin{array}{ll}
C_{1} & C_{2}
\end{array}\right]
\end{array}
$$

Proposição $1 \mathrm{Se}(C, A, E)$ é fortemente detectável e (34) é verificada, então existe uma matriz $T=\left[\begin{array}{cc}T_{1} & T_{2}\end{array}\right] \in$ $\Re^{(q-p) \times n}$, com posto $\left(T E=\left[\begin{array}{ll}T_{1} & 0\end{array}\right]\right)=(q-p)$, tal que o subespaço $\mathcal{V}=\operatorname{Im}\left[\begin{array}{l}V_{1} \\ V_{2}\end{array}\right]$, $\operatorname{com} T E V=T_{1} V_{1}=0 \mathrm{e}$ posto $\left(E V=V_{1}\right)=p$, é $(C, A, E)$-externamente detectável e posto $\left(\left[\begin{array}{c}T E \\ L_{\infty} A \\ C\end{array}\right]=\left[\begin{array}{cc}T_{1} & 0 \\ J_{3} & J_{4} \\ C_{1} & C_{2}\end{array}\right]\right)=n$.

A demonstração do resultado anterior baseia-se numa seqüência particular de transformações de coordenadas que permite construir soluções para o Passo 1 do Algoritmo 1 , independentemente da regularidade do par $(E, A)$ (veja (Castelan and da Silva, 2005)):

\section{Algoritmo 2}

Passo 1: Se as matrizes do sistema não estiverem na forma (35), encontre $Q=\left[\begin{array}{l}Q_{1}^{\prime} \\ Q_{2}^{\prime}\end{array}\right] \in \Re^{n \times n}$ e $R=$ $\left[\begin{array}{ll}R_{1} & R_{2}\end{array}\right] \in \Re^{n \times n}$, tais que $Q E R=\left[\begin{array}{cc}I_{q} & 0 \\ 0 & 0\end{array}\right]$, e defina: $\left[\begin{array}{ll}J_{1} & J_{2} \\ J_{3} & J_{4}\end{array}\right]=Q A R$ e $\left[\begin{array}{ll}C_{1} & C_{2}\end{array}\right]=C R$. Senão: $Q=R=I_{n}$.

Passo 2: Encontre uma matriz inversível $\bar{R}=\left[\begin{array}{c}\bar{R}_{1} \\ \bar{R}_{2}\end{array}\right]$, tal que: $\left[\begin{array}{c}\bar{R}_{1} \\ \bar{R}_{2}\end{array}\right]\left[\begin{array}{l}J_{4} \\ C_{2}\end{array}\right]=\left[\begin{array}{c}\bar{J}_{4} \\ 0\end{array}\right], \operatorname{posto}\left(\bar{J}_{4}\right)=n-q$. Considere $\left[\begin{array}{c}\bar{J}_{3} \\ \bar{C}_{1}\end{array}\right]=\left[\begin{array}{c}\bar{R}_{1} \\ \bar{R}_{2}\end{array}\right]\left[\begin{array}{c}J_{3} \\ C_{1}\end{array}\right]$ e defina o sistema auxiliar representado por $(\bar{C}, \bar{A}, \bar{E})$, em que: $\bar{E}=\left[\begin{array}{cc}I_{q} & 0 \\ 0 & 0\end{array}\right]$, $\bar{A}=\left[\begin{array}{ll}J_{1} & J_{2} \\ \bar{J}_{3} & \bar{J}_{4}\end{array}\right]$ e $\bar{C}=\left[\begin{array}{ll}\bar{C}_{1} & 0\end{array}\right]$. 
Passo 3: Encontre $M=\left[\begin{array}{cc}M_{1} & M_{2}\end{array}\right] \in \Re^{q \times q}$ tal que $\bar{C}_{1}\left[\begin{array}{ll}M_{1} & M_{2}\end{array}\right]=\left[\begin{array}{ll}\tilde{C}_{1} & 0\end{array}\right], \operatorname{com} \tilde{C}_{1} \in \Re^{p \times p} \mathrm{de}$ posto completo. Calcule $\bar{M}=\left[\begin{array}{cc}M_{3} & M_{4}\end{array}\right] \in \Re^{(n-q) \times q}$ que verifica $\bar{J}_{4}\left[\begin{array}{ll}M_{3} & M_{4}\end{array}\right]=-\bar{J}_{3}\left[\begin{array}{ll}M_{1} & M_{2}\end{array}\right]$. Seja $\tilde{M}=\left[\begin{array}{c}\tilde{M}_{1}^{\prime} \\ \tilde{M}_{2}^{\prime}\end{array}\right] \in \Re^{n-q \times n-q}$ a inversa de $M$ : $\left[\begin{array}{ll}M_{1} & M_{2}\end{array}\right]\left[\begin{array}{c}\tilde{M}_{1}^{\prime} \\ \tilde{M}_{2}^{\prime}\end{array}\right]=I_{q}$. Considere as matrizes nãosingulares

$$
\tilde{Q}=\left[\begin{array}{cc}
\tilde{M}_{1}^{\prime} & 0 \\
\tilde{M}_{2}^{\prime} & 0 \\
0 & I_{n-q}
\end{array}\right] \text { e } \tilde{P}=\left[\begin{array}{ccc}
M_{1} & M_{2} & 0 \\
M_{3} & M_{4} & I_{n-q}
\end{array}\right]
$$

e obtenha o sistema representado por $(\tilde{E}, \tilde{A}, \tilde{C})$ :

$$
\begin{aligned}
& \tilde{E}=\tilde{Q} \bar{E} \tilde{P}=\left[\begin{array}{ccc}
I_{p} & 0 & 0 \\
0 & I_{q-p} & 0 \\
0 & 0 & 0
\end{array}\right], \tilde{C}=\bar{C} \tilde{P}=\left[\begin{array}{c}
\tilde{C}_{1}^{\prime} \\
0 \\
0
\end{array}\right]^{\prime}, \\
& \tilde{A}=\tilde{Q} \bar{A} \tilde{P}=\left[\begin{array}{ccc}
\tilde{J}_{11} & \tilde{J}_{12} & \tilde{J}_{13} \\
\tilde{J}_{21} & \tilde{J}_{22} & \tilde{J}_{23} \\
0 & 0 & \tilde{J}_{33}
\end{array}\right] \begin{array}{l}
\tilde{J}_{12} \in \Re^{p \times(q-p)} \\
\tilde{J}_{22} \in \Re^{(q-p) \times(q-p)} \\
\tilde{J}_{33}=\bar{J}_{4}
\end{array}
\end{aligned}
$$

Passo 4: Encontre uma solução para a equação de Sylvester de ordem reduzida

$$
\tilde{T}_{12} \tilde{J}_{22}-H_{T} \tilde{T}_{12}=-\tilde{T}_{11} \tilde{J}_{12}, \sigma\left(H_{T}\right)=\Lambda_{T} \subset \mathcal{C}^{-}
$$

na qual $H_{T} \in \Re^{(q-p) \times(q-p)}, \tilde{T}_{11} \in \Re^{(q-p) \times p}$ e $\tilde{T}_{12} \in$ $\Re^{(q-p) \times(q-p)}$ deve ser não-singular.

Passo 5: Calcule: $T_{1}=\left[\begin{array}{ll}\tilde{T}_{11} & \tilde{T}_{12}\end{array}\right]\left[\begin{array}{c}\tilde{M}_{1}^{\prime} \\ \tilde{M}_{2}^{\prime}\end{array}\right]$. Na base original, tem-se: $T E=\left[\begin{array}{ll}T_{1} & 0\end{array}\right] Q$.

Observe que a escolha das matrizes $Q, R, \bar{R}$ e $M$ não é única. Então, elas devem ser definidas como matrizes ortogonais, o que contribui para a estabilidade e eficiência numérica da implementação computacional do algoritmo. Além disso, podese verificar que, por construção, o sistema normal de ordem reduzida $(q-p)$ representado por $\left(\tilde{J}_{12}, \tilde{J}_{22}\right)$ é detectável. Portanto, é sempre possível obter uma solução adequada para a equação de Sylvester (36) (Chen, 1996). Finalmente, salientamos que diferentes técnicas podem ser utilizadas para a resolução do Passo 4. Dentre estas, a resolução de uma desigualdade de tipo Lyapunov associada ao sistema normal de ordem reduzida é mostrada na seção seguinte.

\section{ESTABILIZAÇÃO VIA RELAÇÕES DE TIPO LYAPUNOV}

Como em (Castelan et al., 2003), soluções para o Passo 4 do Algoritmo 2 podem ser obtidas a partir de uma desigual- dade matricial linear (LMI) associada ao sistema de ordem reduzida representado pelo par $\left(\tilde{J}_{12}, \tilde{J}_{22}\right)$. Por hipótese, o sistema de ordem reduzida é detectável e $p<q$. Assim, considere matrizes $S_{21} \in \Re^{(q-p) \times p}$ e $S_{22} \in \Re^{(q-p) \times(q-p)}$, com $S_{22}=S_{22}^{\prime}>0$, soluções da LMI de ordem reduzida seguinte:

$$
\tilde{J}_{22}^{\prime} S_{22}+S_{22} \tilde{J}_{22}+\tilde{J}_{12}^{\prime} S_{21}^{\prime}+S_{21} \tilde{J}_{12}<0
$$

Seja $L_{2} \in \Re^{(q-p) \times p}$ tal que:

$$
S_{22} L_{2}=S_{21}
$$

Por construção, $\sigma\left(\tilde{J}_{22}+L_{2} \tilde{J}_{12}\right) \in \mathcal{C}^{-}$. Então, para qualquer matriz não-singular $\tilde{T}_{12} \in \Re^{(q-p) \times(q-p)}$, pode-se definir a matriz $H_{T} \in \Re^{(q-p) \times(q-p)}$ que verifica $\sigma\left(H_{T}\right)=$ $\sigma\left(\tilde{J}_{22}+L_{2} \tilde{J}_{12}\right) \in \mathcal{C}^{-}$, a partir da relação de similaridade $\tilde{T}_{12}\left(\tilde{J}_{22}+L_{2} \tilde{J}_{12}\right)=H_{T} \tilde{T}_{12}$. A matriz $\tilde{T}_{11}$ correspondente, que satisfaz (36), verifica:

$$
\tilde{T}_{11}=\tilde{T}_{12} L_{2}
$$

O desenvolvimento anterior mostrou que a solução da LMI (37) implica na solução da equação de Sylvester de ordem reduzida (36). Também é possível mostrar o caminho oposto. Isto implica que não há perda de generalidade ao se considerar a solução do Passo 4 do Algoritmo 2 via a LMI (37). Relembre que o Algoritmo 2 pode ser utilizado para a realização do Passo 1 do Algoritmo 1. Além disso, sob a condição de acoplamento (16), a equação de Sylvester (14) envolvida no Passo 2 desse primeiro algoritmo é equivalente as relações (21)-(23) do Teorema 2.

Observação 4 Como $\tilde{T}_{12}$ é uma matriz invertível qualquer, é possível arbitrar $\tilde{T}_{12}=S_{22}$. Deste modo, a relação seguinte pode ser utilizada para o cálculo de $V=\left[\begin{array}{l}V_{1} \\ V_{2}\end{array}\right]$ tal que $T E V=T_{1} V_{1}=0$ :

$$
\operatorname{Ker}\left(T_{1}\right)=\operatorname{Ker}\left(\left[\begin{array}{ll}
S_{21} & S_{22}
\end{array}\right]\left[\begin{array}{c}
\tilde{M}_{1}^{\prime} \\
\tilde{M}_{2}^{\prime}
\end{array}\right]\right)
$$

Baseado nos resultados e comentário anteriores, o procedimento básico seguinte pode ser usado para calcular uma matriz de realimentação de saídas que estabiliza fortemente o sistema em malha fechada quando a condição $(m+p)>q+d$ é satisfeita. Este algoritmo pode ser visto como uma extensão do algoritmo proposto em (Castelan et al., 2003) para a estabilização de sistemas normais e também generaliza resultados anteriores propostos em (Llanos Villarreal et al., 2000; Castelan et al., 2002) para classes particulares de sistemas descritores. 


\section{Algoritmo 3}

\section{Passo 1}

1.1) Utilizar os Passos 1 a 3 do Algoritmo 2 para encontrar as matrizes $\tilde{J}_{12}$ e $\tilde{J}_{22}$.

1.2) Resolver a $L M I$ de ordem reduzida (37) para encontrar $S_{12}$ e $S_{22}=S_{22}^{\prime}>0$.

1.3) Calcular $T_{1}=\left[\begin{array}{ll}S_{11} & S_{12}\end{array}\right]\left[\begin{array}{c}\tilde{M}_{1}^{\prime} \\ \tilde{M}_{2}^{\prime}\end{array}\right]$. Sejam as matrizes $\mathcal{A}=J_{1}, \mathcal{B}=\left[-J_{2} 0\right], \mathcal{C}=\left[\begin{array}{l}-J_{3} \\ -T_{1}\end{array}\right]$ e $\mathcal{D}=$ $\left[\begin{array}{cc}-J_{4} & 0 \\ 0 & 0\end{array}\right] . \mathrm{Se}(\mathcal{A}, \mathcal{B}, \mathcal{C}, \mathcal{D})$ não tem zeros invariantes, então ir ao Passo 3; caso contrário, repetir o Passo 1 usando uma nova decomposição de $\bar{C}_{1}$.

\section{Passo 2}

2.1) Calcular $V=\left[\begin{array}{l}V_{1} \\ V_{2}\end{array}\right]$ que verifique $T E V=T_{1} V_{1}=0$ e posto $(C V)=p$.

2.2) Resolver a equação (21), sob as restrições (22) e (23), e encontrar as matrizes $P, Y, \bar{P}$ e $W_{\Pi}$.

Passo 3 A matriz de realimentação de saídas verifica: $K C P=Y \longleftrightarrow K C V \bar{P}=W_{\Pi}$, se $V^{\prime} V=I_{p}$.

Esta seção será finalizada com algumas observações relativas ao algoritmo anterior. Estas observações complementam os comentários e observações já realizados em relação aos Algoritmos 1 e 2 (veja também (Castelan et al., 2003)).

Observação 5 O teste de existência de zeros invariantes realizado no final do Passo 1 tem por objetivo evitar a parada do algoritmo nos casos raros em que uma solução particular torne o Passo 2 não viável. Então, de maneira similar à estratégia utilizada no Algoritmo 1, a definição de uma nova decomposição de $\bar{C}_{1}$ objetiva encontrar uma nova solução $\left(S_{21}, S_{22}\right)$ que viabilize o Passo 2. Além disso, a definição de $\mathcal{A}, \mathcal{B}, \mathcal{C}$ e $\mathcal{D}$ permite utilizar neste teste as rotinas existentes para o cálculo de zeros invariantes de sistemas normais.

Observação 6 No Passo 2, após o cálculo de $V=$ $\left[\begin{array}{ll}V_{1}^{\prime} & V_{2}^{\prime}\end{array}\right]^{\prime}$, objetiva-se resolver as condições (21), (22) e (23), Como $V_{2} \in \Re^{(n-q) \times p}$ pode ser escolhida livremente, pode-se adotar a seguinte estratégia para garantir que posto $\left(C V=C_{1} V_{1}+C_{2} V_{2}\right)=p$, o que garante o cálculo de $K$ a partir das relações no Passo 3 :

- se posto $\left(C_{1} V_{1}\right)=p$, então considera-se $V_{2}=0$;

- se posto $\left(C_{1} V_{1}\right)<p$, então $V_{2} \neq 0$ deve garantir que o posto $(C V)$ seja completo.
Observação 7 No presente artigo, a estabilidade assintótica é, conjuntamente com a regularidade e a ausência de modos impulsivos, um dos requerimentos para o sistema em malhafechada. Cabe salientar que os passos do algoritmo acima podem ser adaptados para obter posicionamento de pólos em regiões do tipo LMI e, em particular, para tratar o problema de estabilização forte de sistemas descritores em tempo discreto. Para tanto, deve-se substituir as condições de estabilidade expressas por (19) e (20), pelas condições propostas em, por exemplo,(Chilali and Gahinet, 1996) para caracterizar o posicionamento regional dos autovalores de $H_{V}$ e $H_{T}$ (veja também (Castelan et al., 2002; Castelan and da Silva, 2005)).

Observação 8 Pacotes computacionais apropriados para a resolução numérica de LMIs/LMEs podem ser utilizados para encontrar soluções factíveis para as relações de tipo Lyapunov envolvidas nos dois passos do algoritmo (Boyd et al., 1994).

\section{EXEMPLO}

Considere o sistema descritor representado pelas matrizes (Chu et al., 1999):

$$
\begin{gathered}
E=\left[\begin{array}{rrlll}
0.00 & 0.00 & -0.82 & 0.00 & 0.00 \\
0.00 & 0.00 & 0.00 & 0.00 & 0.00 \\
0.00 & 0.00 & 0.00 & 0.00 & 0.00 \\
1.72 & 0.00 & 0.00 & 0.00 & 0.00 \\
0.00 & 0.00 & 0.00 & 0.00 & 1.00
\end{array}\right] \\
A=\left[\begin{array}{rrrrr}
0.00 & 0.00 & 1.23 & 0.00 & 0.00 \\
1.10 & 0.00 & 0.00 & 0.00 & 0.00 \\
0.00 & 1.56 & 0.00 & 0.00 & 1.01 \\
0.00 & 0.00 & 1.98 & 0.00 & 0.00 \\
0.00 & 0.00 & 0.00 & 0.00 & 0.00
\end{array}\right] \\
B=\left[\begin{array}{rrrr}
0.41 & -6.64 & -2.34 \\
0.00 & 0.00 & 0.45 \\
0.00 & 0.00 & 0.00 \\
1.72 & -15.48 & 0.00 \\
7.00 & 0.00 & -1.00
\end{array}\right], C^{\prime}=\left[\begin{array}{rr}
-1 & 0 \\
0 & 0 \\
0 & 0 \\
-1 & 1 \\
0 & 1
\end{array}\right]
\end{gathered}
$$

$\mathrm{O} \operatorname{par}(E, A)$ é não-regular, com $d=\operatorname{dim}\{\operatorname{Ker}(E) \cap$ $\operatorname{Ker}(A)\}=1$. Os pólos finitos são dados por: $\sigma(E, A)=$ $\{0.0,-1.5\}$. O sistema $(C, E, A, B)$ é fortemente controlável e fortemente detectável, e $m+p=5>q+d$. Pode-se então aplicar o Algoritmo 3 como segue ${ }^{2}$.

As matrizes do sistema de ordem reduzida no Passo

1.1 correspondem a: $\left[\begin{array}{c}\tilde{J}_{12} \\ \ldots \\ \tilde{J}_{22}\end{array}\right]=\left[\begin{array}{c}-0.5586 \\ -1.0052 \\ \ldots \\ -1.5000\end{array}\right]$.

${ }^{2}$ Utilizou-se o software SCILAB, desenvolvido no INRIA/França. 


\begin{tabular}{|c|c|c|c|}
\hline Passo 2.2 & $\alpha=0$ & $\alpha=2$ & $\alpha=4$ \\
\hline $\bar{P}$ & $\left.\begin{array}{rr}11653.916 & -2175.534 \\
-2175.534 & 13690.257\end{array}\right]$ & {$\left[\begin{array}{rr}778624.240 & -47603.884 \\
-47603.884 & 771411.610\end{array}\right]$} & {$\left[\begin{array}{cc}5243.466 & 2874.604 \\
2874.6048 & 16237.186\end{array}\right]$} \\
\hline$K$ & {$\left[\begin{array}{rr}0.8174 & -0.2826 \\
-0.3254 & 0.0654 \\
2.4444 & 0.0000\end{array}\right]$} & {$\left[\begin{array}{rr}0.8453 & -0.5934 \\
-0.3133 & -0.0693 \\
2.4444 & 0.0000\end{array}\right]$} & {$\left[\begin{array}{rr}1.5118 & -1.3365 \\
-0.0240 & -0.3919 \\
2.4444 & 0.0000\end{array}\right]$} \\
\hline$\sigma(E, A+B K C)$ & $\{\underbrace{-3.8993}, \underbrace{-1.6629 \pm 1.3894 i}\}$ & $\{\underbrace{-3.8993}, \underbrace{-2.7098 \pm 1.7896 i}\}$ & $\{\underbrace{-3.8993}, \underbrace{-4.3425 \pm 2.1982 i}\}$ \\
\hline & Passo1 Passo2 & Passo1 & Passo1 \\
\hline
\end{tabular}

Tabela 1: Diferentes soluções usando posicionamento regional de pólos no Passo 2.2

Uma solução para o Passo 1.2 é: $\left[\begin{array}{lll}S_{21} & \vdots & S_{22}\end{array}\right]=$ $\left[\begin{array}{llll}0.6213 & 0.3455 & \vdots & 0.2894\end{array}\right]$, a qual resulta no passo $1.3 \mathrm{em:} \quad T_{1}=\left[\begin{array}{ll}S_{21} & S_{22}\end{array}\right]\left[\begin{array}{l}\bar{M}_{1}^{\prime} \\ \bar{M}_{2}^{\prime}\end{array}\right]=$ $\left[\begin{array}{lll}-0.6038 & 0.3753 & 0.2894\end{array}\right]$. Esta solução torna viável o Passo 2, pois não implica na existência de zeros invariantes. Determina-se, então:

$$
\left[\begin{array}{l}
V_{1} \\
V_{2}
\end{array}\right]=\left[\begin{array}{rrrrr}
0.3770 & -0.1032 & 0.9204 & 0 & 0 \\
0.4889 & 0.8662 & -0.1032 & 0 & 0
\end{array}\right]^{\prime}
$$

A tabela 1 mostra, em particular, diferentes soluções obtidas a partir do Passo 2.2. Buscou-se, neste passo, soluções factíveis para (21), sob as restrições (22) e (23), que posicionam os dois pólos finitos associados com partes reais tais que $\operatorname{Re}\left(\lambda_{i}\right)<-\alpha$, para $\alpha=0,2$ e 4 . Para a obtenção deste tipo de posicionamento regional de pólos, basta substituir $A$ por $(A+\alpha E)$ em (21). Note que as três matrizes de realimentação de saídas obtidas são tais que o autovalor -3.8993, correspondente ao Passo 1, faz parte do conjunto de pólos finitos do sistema em malha fechada. Como o número de pólos finitos é igual a 3, as três soluções propostas estabilizam fortemente o sistema em malha-fechada.

\section{CONCLUSÃO}

Considerou-se o problema de cálculo de uma matriz de realimentação de saídas que estabiliza fortemente um sistema descritor. Condições necessárias e suficientes para a solução do problema foram obtidas sob a forma de equações acopladas de Sylvester e de equações acopladas de Lyapunov. Além disso, mostrou-se que os subespaços fortemente O.S. $(C, A, E, B)$-invariantes têm um papel fundamental na estabilização usando realimentação de saídas em sistemas descritores.
Foram apresentados algoritmos para o cálculo da matriz de realimentação de saídas, baseados em técnicas de posicionamento de autoestrutura ou em técnicas para a solução de LMI/LMEs. Estes algoritmos podem ser aplicados a sistemas descritores que satisfazem a condição $(m+p)>q+d$, a qual pode ser considerada como uma generalização da condição de Kimura. É importante observar que os algoritmos propostos também podem ser utilizados para a estabilização forte de sistemas para os quais esta condição não é satisfeita, utilizando-se compensadores dinâmicos clássicos de ordem $n_{c}>q+d-(m-p)$. Cabe também salientar a flexibilidade oferecida pela abordagem baseada nas equações de tipo Lyapunov, a qual pode ser adaptada para considerar outros requisitos e, particularmente, para a obtenção de posicionamento regional de pólos. Desta forma, os resultados apresentados generalizaram resultados obtidos anteriormente para o caso de sistemas normais (Castelan et al., 2003) e para classes particulares de sistemas descritores (Llanos Villarreal et al., 2000; Castelan et al., 2002)

Entretanto, a aplicação da abordagem apresentada na solução de outros problemas de controle de sistemas descritores por realimentação estática de saídas constitui-se num campo de possível investigação, no qual pode-se destacar: $i$ ) a consideração de requisitos de desempenho e de robustez; ii) o uso das tranformações de coordenadas do Algoritmo 2 para a realização de posicionamento arbitrário de pólos em sistemas que satisfaçam a condição $m . p \geq q$, como extensão da abordagem paramétrica proposta em (Alexandridis and Paraskevopoulos, 1996); e iii) a generalização dos conceitos e resultados apresentados para o caso de sistemas descritores retangulares, i.e.: $E \in \Re^{m \times n}$, com $m \neq n$ (Ishihara and Terra, 2001; Hou, 2004). 


\section{AGRADECIMENTOS}

Este trabalho foi realizado com o apoio financeiro do $\mathrm{CNPq}$ e da CAPES. O autor agradece ao editor associado e aos revisores por seus comentários e sugestões.

\section{REFERÊNCIAS}

Alexandridis, A. T. and Paraskevopoulos, P. N. (1996). A new approach to eigenstruture assignment by output feedback, IEEE Transactions on Automatic Control 41(7): 1046-1050.

Barbosa, K. A. and Trofino, A. (2003). Síntese $h_{\infty}$ para sistemas com restrições algébricas no estado, Revista Controle e Automação 14(3): 254-261.

Boyd, S. P., El Ghaoui, L., Feron, E. and Balakrishhnan, V. (1994). Linear Matrix Inequalities in System and Control Theory, Philadelphia SIAM.

Bunse-Gerstner, A., Mehrmann, V. and Nichols, N. K. (1992). Regularization of descriptor systems by derivative and proportional state feedback, Siam J. of Matrix Analysis and Applications 13(1): 46-67.

Castelan, E. B. and da Silva, V. G. (2005). On the solution of a sylvester equation appearing in descriptor systems control theory, Systems and Control Letters 54(2): 109117.

Castelan, E. B., Hennet, J. C. and Llanos Villarreal, E. R. (2003). Quadratic characterization and use of output stabilizable subspaces, IEEE Transactions on Automatic Control 48(4): 654-660.

Castelan, E. B., Llanos Villarreal, E. R. and Tarbouriech, S. (2001). Quadratic characterization and use of output stabilizable subspaces in descriptor systems, Proceedings of the 1st IFAC Symposium on System Structure and Control, Prague, p. 5.

Castelan, E. B., Silva, A. S., Llanos Villarreal, E. R. and Tarbouriech, S. (2002). Regional pole placement by output feedback for a class of descriptor systems, Proceedings of the 15th Triennial World Congress of IFAC, (IFAC'02), Barcelona.

Chen, C. T. (1996). Linear System Theory ans Design, Holt, Rinehart and Winston.

Chilali, M. and Gahinet, P. (1996). $h_{\infty}$ design with pole placement constraints: An lmi approach, IEEE Trans. Automatic Control 41(03): 358-367.
Chu, D. (2001). A case study for the open question: Disturbance decoupling problem for singular systems by output feedback, IEEE Transactions on Automatic Control 46(12): 1924-1930.

Chu, D. L., Chan, H. C. and Ho, D. W. C. (1999). Necessary and suficient conditions for the output feedback regularization of descriptor systems, IEEE Transactions on Automatic Control 44(2): 405-412.

Dai, L. (1989). Singular Control System, Springer-Verlag.

Duan, G. R. (1995). Parametric eigenstructure assignment in descriptor systems via output feedback, IEE Proceedings on Control Theory Theory and Applications 142(6): 611-616.

Duan, G. R. (1999). Eigenstructure assignment in descriptor systems via output feedback: A new complete parametric approach, International Journal of Control 72(4): 345-364.

Duan, G. R. (2004). Parametric eigenstructure assignment in second-order descriptor linear systems, IEEE Transactions on Automatic Control 49(10): 1789-1795.

Duan, G. R., Liu, G. P. and Thompson, S. (2000). Disturbance decoupling in descriptor systems via output feedback - a parametric eigenstructure assignment approach, Proceedings of the 39th Conference on Decision and Control, Sydney, pp. 3660-3665.

Fletcher, L. R. (1988). Eigenstructure assingment by output feedback in descriptor systems, IEE Proceedings on Control Systems Theory and Applications 135(4): 302308.

Hou, M. (2004). Controllability and elimination of impulsive modes in descriptor systems, IEEE Transactions on Automatic Control 49(10): 1723-1727.

Ishihara, J. Y. and Terra, M. H. (2001). Impulse controllability and observability of rectangular descriptor systems, IEEE Transactions on Automatic Control 46(6): 991994.

Kuo, C. H. and Fang, C. H. (2003). An lmi approach to admissibilization of uncertain descriptor systems via static output feedback, Proceedings of the American Control Conference, Denver, pp. 5104-5109.

Lewis, F. L. (1986). A survey of linear singular systems, Circuits, Systems and Signal Processing 5(1): 3-36.

Llanos Villarreal, E. R., Castelan, E. B. and Silva, A. S. (2000). Output feedback for stabilization of a class descriptor systems, Anais do XIII Congresso Brasileiro de Automática, Florianópolis, Brasil, pp. 423-428. 
Ozcaldiran, K. S. and Lewis, F. L. (1990). On the regularizability of singular systems, IEEE Trans. Automatic Control 35(10): 1156-1160.

Syrmos, V. L. and Lewis, F. L. (1993). Output feedback eigenstructure assignment using two sylvester equations, IEEE Transactions on Automatic Control 38(3): 495499.

Varga, A. (1995). On stabilization methods of descriptor systems, Systems and Control Letters 24(2): 133-138.

Verghese, G. C., Lèvy, B. and Kailath, T. (1981). A general state space for singular systems, IEEE Transactions on Automatic Control 26: 811-831.

Zhang, G., Zhang, Q., Chen, T. and Lin, Y. (2005). On lyapunov theorems for descriptor systems, Dynamics of Continuous, Discrete and Impulsive Systems - Series B: Applications and Algorithms 10(5): 709-726. 